\title{
Penetration of a resin-based filling material into lateral root canals and quality of obturation by different techniques
}

\section{André Luiz da Costa MICHELOTTO(a) Cacio MOURA-NETTO(b) Angela Toshie ARAKI (b) Eduardo AKISUE(c) Gilson Blitzkow SYDNEY(d)}

(a) Universidade Tuiuti do Paraná - UTP, School of Dentistry, Curitiba, PR, Brazil.

(b) Universidade Cruzeiro do Sul, School of Dentistry, Graduate Program in Dentistry, São Paulo, SP, Brazil.

(c) Universidade Santa Cecilia - Unisanta, School of Dentistry, Santos, SP, Brazil.

(d) Universidade Federal do Paraná, School of Dentistry, Curitiba, PR, Brazil.

Declaration of Interests: The authors certify that they have no commercial or associative interest that represents a conflict of interest in connection with the manuscript.

Corresponding Author:

Cacio Moura-Netto

E-mail: caciomn@usp.br

DOI: 10.1590/1807-3107BOR-2015.vol29.0010

Submitted: May 09, 2014

Accepted for publication: Sep 08, 2014

Last revision: Oct 17, 2014

\begin{abstract}
The aim of this study was to evaluate the penetration of a resin/polyester polymer-based material (Resilon Real Seal; SybronEndo Corp., Orange, USA) into simulated lateral canals, and the quality of obturations by different techniques. A total of 30 standardized simulated canals were divided into three groups according to the technique of obturation used: MS (McSpadden), SB (SystemB/Obtura II), and LC (Lateral Condensation). To analyze the penetration of the filling material, the simulated canals were digitalized and the images were analyzed using the Leica QWIN Pro v2.3 software. The data of the middle and apical thirds were separately submitted to analysis of variance (ANOVA), followed by the Tukey's test for the comparison of the techniques. Results showed a significant difference $(p<0.05)$ between groups (LC < SB) in the middle third, and a significant difference $(p<0.05)$ between groups (LC $<$ SB and MS $<\mathrm{SB}$ ) in the apical third. To analyze the quality of the obturations, the canals were radiographed and evaluated by three examiners. The Kappa test on interexaminer agreement and the nonparametric Kruskal-Wallis test indicated no significant difference between filling techniques. It was concluded that Resilon achieves greater levels of penetration when associated with thermoplastic obturation techniques.
\end{abstract}

Keywords: Root Canal Filling Materials; Root Canal Obturation; Endodontics.

\section{Introduction}

The goal of endodontic therapy is to obtain a root canal filling that allows tridimensional sealing of the root canal system, using a nonirritant material to support periapical healing. The root canal system has a very complex morphology with many irregularities including fins, deltas, and accessory and lateral canals. Lateral canals are reported in $27.4-45 \%$ of all teeth, ${ }^{2,3}$ and majority are located in the apical third of the roots. ${ }^{3,4}$ Reports have shown developing or persistent periapical pathology around untreated and/or unfilled lateral canals. ${ }^{5,6}$ Thus, filling these areas appears to be very important to improve the success rate of endodontic therapy.

Obturation techniques involving gutta-percha plasticization have shown the greatest efficiency in filling root canal systems. ${ }^{7,8}$ An alterna- 
tive is the Resilon cone (Resilon Research, LLC, North Branford, USA), which is a resin/polyester polymerbased obturation material associated with a hydrophilic resin-based sealer (Real Seal, SybronEndo Corp., Orange, USA). This obturation system was introduced with the concept of monoblock formation between the cone and sealer. The hydrophilic property of this methacrylate resin-based sealer allows deeper penetration into dentinal tubules, which may increase its sealing ability. ${ }^{9}$

According to the manufacturer, the polymers in Resilon (Resilon Research, LLC) provide the material with thermoplastic properties allowing it to be applied using thermoplastic techniques, ${ }^{10,11,12}$ because its melting point is the same as gutta-percha $\left(60^{\circ} \mathrm{C}\right) .{ }^{13}$ This could be an advantage because several studies showed superior sealing ability and filling material adaptation with a thermoplastic technique.,14

Thus, the aim of this study was to evaluate the penetration of Resilon (Real Seal, SybronEndo Corp., Orange, USA) into simulated lateral canals, and the quality of the fillings obtained using different techniques: Lateral Condensation (LC), System B/Obtura II (SB), and McSpadden (MS).

\section{Methodology}

This study used 30 simulated curved canals (angle: approximately $30^{\circ}$; length: $24 \mathrm{~mm}$ ), prepared at the Prosthesis Laboratory, Dental School, Universidade
Federal do Paraná (UFPR, Curitiba, Brazil) ready for obturation. Each sample contained four lateral canals (diameter: $0.3 \mathrm{~mm}$; length: $7.0 \mathrm{~mm}$ ), two located in the middle and two in the apical third (Figure 1). The specimens were divided into three groups according to the filling technique: MS, SB, and LC Groups.

\section{Filling protocol}

The filling material used was the Real Seal system (SybronEndo Corp., Orange, USA). For all groups, a 0.04-taper Resilon cone was fitted to the working length ( $1 \mathrm{~mm}$ prior to canal exit). After primer application, the sealer was inserted into the canal using the Resilon cone, until the sealer covered the entire extension of the cone. These procedures were performed according to the manufacturer's instructions.

\section{Group MS (McSpadden)}

A thermo compactor (SybronEndo Corp.) with a larger tip (\#60), attached to a contra-angle handpiece and a low-speed motor, was inserted into the filled canal. The compactor was activated at 15,000 rpm and inserted into the canal with smooth in and out movements (amplitude: $3 \mathrm{~mm}$ ). The compactor reached $20 \mathrm{~mm}$, at the beginning point of curvature. At this point, the compactor was gently removed from the canal using the same in and out movement. Once thermo-compaction was performed, a cold plugger

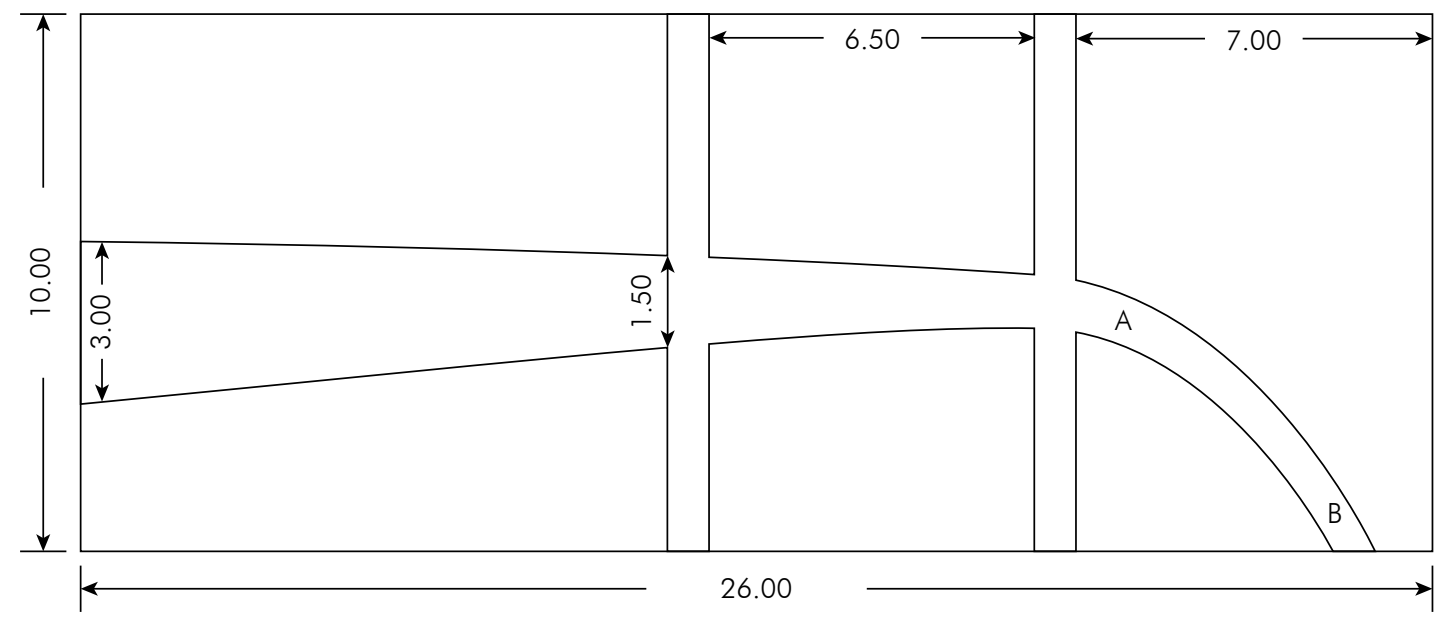

Figure 1. Drawing of an artificial simulated root canal showing lateral canals in the middle and apical thirds. 
was used for vertical condensation of the filling material, to achieve better adaptation to the canal walls.

\section{Group SB (System B/Obtura II)}

The System B device (SybronEndo Corp.) using Resilon pellets (Real Seal, SybronEndo Corp.) was calibrated to $150^{\circ} \mathrm{C}$. After the root canal was filled with the sealer and the solid material (as described above), the \#2 condenser was activated and introduced until the same depth established for the MS group (20.0 $\mathrm{mm}$ ). At this point, heating was stopped and the condenser remained under pressure for 10 seconds. Furthermore, heating was rapidly activated and the condenser was removed from the interior of the canal. Condensation of the remaining thermoplasticized material was performed in the apical third with a cold plugger. Filling of the middle and cervical thirds was completed with successive stages of injection of thermoplasticized Resilon using Obtura II (Obtura Corp., Fenton, USA) and cold vertical condensation using cold pluggers.

\section{Group LC (Lateral Condensation)}

The master Resilon cone was fitted into the root canal with the sealer. Furthermore, spaces were created using a $C$ finger spreader (Dentsply Maillefer, Tulsa, USA) to introduce MF accessory cones until the canal is filled. Obturation was performed with a heated plugger, the excess of gutta-percha was removed, and a cold plugger was used for vertical condensation of the filling material.

\section{Evaluation of penetration of the filling material}

The penetration of the filling material into lateral canals was evaluated using images of the simulated canals taken with a stereomicroscope (Leica Microsystems, Bannockburn, USA), and analyzed using the Leica QWIN Pro software (Leica Microsystems). The parameters were the total length of the lateral root canals and the extent of penetration of the filling material. The data were submitted to analysis of variance (ANOVA) and Tukey's test $(p<0.05)$. Statistical analysis was performed with SPSS 13.0 software (SPSS Inc., Chicago, USA).

\section{Radiographic Quality of Obturation}

The quality of root canal filling was scored on the following modified three-point scale: (1) a good score for homogenous filling obturating the entire prepared root canal, well adapted to the canal wall, with only a few minor areas of relative radiolucency (diameter: $\leq 0.25 \mathrm{~mm}$; without porosity); (2) a satisfactory score for imperfect filling with irregularities of $\leq 1 \mathrm{~mm}$, where the filling may be slightly shorter (diameter: $\leq 0.5 \mathrm{~mm}$ ) than the working length (few porosities); and, (3) a bad score for inadequately filled canals with irregularities (diameter: $>1 \mathrm{~mm}$ ), where the filling diameter may be $>0.5 \mathrm{~mm}$ shorter than the working length (several porosities). Three trained examiners performed the evaluations simultaneously. Examiner reliability was assessed by reevaluation of all specimens on a separate occasion. In case of disagreement, the ultimate decision was reached by consensus. The collected data were submitted for statistical analysis using the Kappa correlation test for interexaminer comparison, and the Kruskal-Wallis nonparametric test for score analysis $(p<0.05)$. Statistical analysis was performed with SPSS 13.0 software (SPSS Inc., Chicago, USA).

\section{Results}

The obturation technique had a significant impact on the filling quality of lateral root canals. In the middle third, filling penetration into lateral canals was shallower in the LC Group than in the SB and MS Groups (Figure 2; $p<0.05$ ). On the other hand, there was no significant difference between the SB and MS groups. In the apical third, greater penetration was obtained for the SB Group than the MS and LC groups (Figure 3; $p<0.05$ ). But there was no significant difference between the MS and LC groups $(p>0.05)$.

The quality of the fillings was analyzed by three experienced endodontists using radiographs of the simulated root canals. Interexaminer agreement analysis produced a kappa value of 0.75 (75\%), indicating a high correlation between the three examiners. Accordingly, a nonparametric Kruskal-Wallis test was performed, and indicated no significant difference between the groups $(p>0.05)$. 


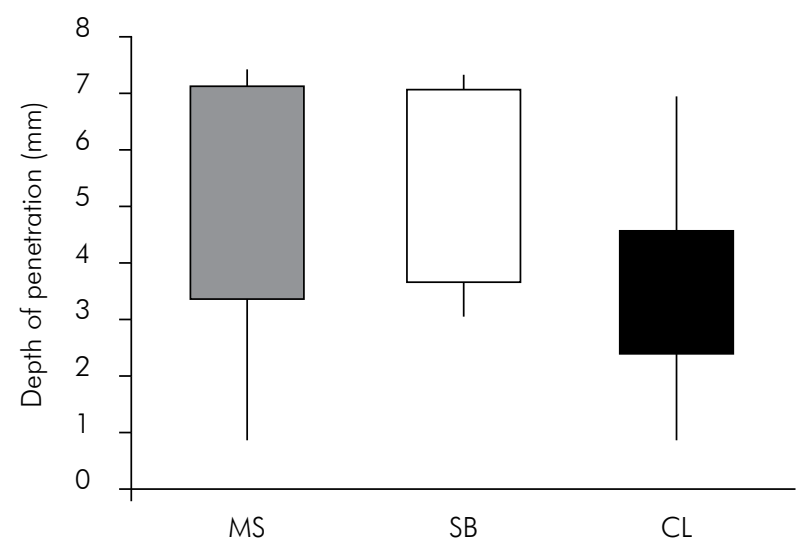

Figure 2. Impact of the obturation technique on filling material penetration $(\mathrm{mm})$ into lateral canals of the middle third region.

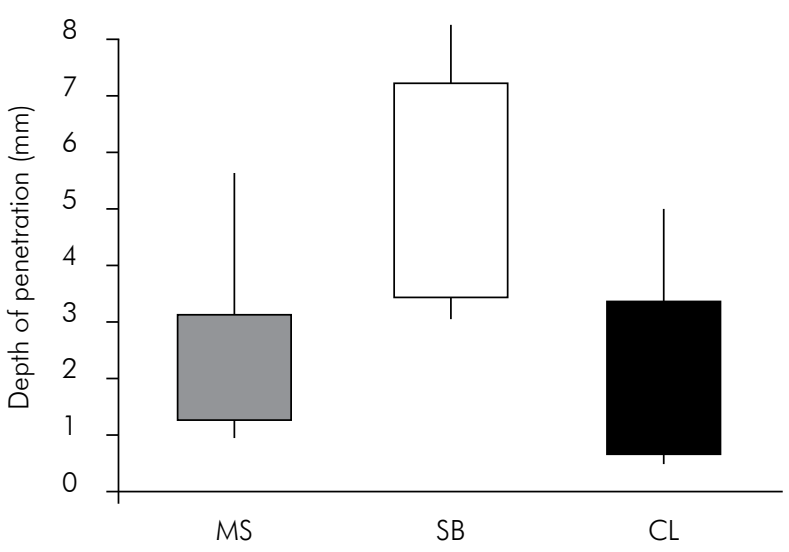

Figure 3. Impact of the obturation technique on filling material penetration $(\mathrm{mm})$ into lateral canals of the apical third region.

\section{Discussion}

The purpose of this study was to compare the efficiency of three obturation techniques for the penetration of the Resilon Real Seal (SybronEndo Corp.) filling material into lateral canals. Standardized experiments were conducted with simulated root canals to avoid the variables between natural teeth. ${ }^{78,15,16}$ These root canals had lateral canals in the middle and apical thirds, visible in the buccolingual direction, to evaluate the penetration depth of filling material by radiographic analysis.

The thermoplastic properties of Resilon are attributed to the addition of polycaprolactone, which has a melting point of $60^{\circ} \mathrm{C}^{12}$ and is indicated for thermoplastic obturation techniques. ${ }^{8,14,17}$ Thermoplastic
Resilon was found superior to conventional guttapercha for plasticization. ${ }^{17,18}$

The present study demonstrates that the relative efficiency of the obturation techniques depends on the position of the lateral root canals. In the middle third, the filling material penetrated deeper by thermoplastic obturation (SB or MS) than by LC. In the apical third, superior obturation was obtained by SB than by MS or LC. In fact, plasticization of filling material by MS was more efficient in the middle third than in the apical third of the root. This discrepancy is consistent with the fact that the thermo compactor was inserted up to the beginning of the curvature. After this point, the filling material had a poorer plasticization; therefore, penetration into the apical lateral canals was lower. This finding suggests that the superior efficacy of MS is limited to the straight portion of the root canal. Beyond the compactor tip, the filling material is poorly plasticized, as previously reported. ${ }^{\text {? }}$ The thermo compactor of MS only remained activated long enough to initiate filling material plasticization. A longer activation time could improve the flowability of filling material but could also increase the porosity of the obturation. ${ }^{7}$ Thus, SB is more effective for the plasticization of filling material in the apical third.

Karr et al. ${ }^{19}$ and Tanomaru-Filho et al. ${ }^{20}$ reported similar flow for Resilon and gutta-percha in lateral grooves and depressions, when obturation was performed using the warm vertical compaction (System B) technique. Karabucak et al..$^{14}$ evaluated the ability of Obtura II and Calamus to fill artificially created lateral canals using standard gutta-percha and Resilon. Their results indicated that the flow of the filling material into the lateral canals depends on the viscoelastic properties of the filling material rather than the mechanical properties of the delivery systems. They suggest that Resilon filling material flows better into lateral canals using a single backfill technique.

The quality of the fillings obtained with the three obturation techniques was analyzed by three experienced endodontists using radiographs of the simulated root canals. The fillings were scored as good (without porosity), satisfactory (few porosities), or bad (several porosities). The three examiners agreed that all three techniques generated similarly satisfactory results regarding the quality of the obturations. 
In addition, similar results were found by others. ${ }^{21,22}$ There are also studies showing satisfactory clinical results ${ }^{9,11}$ and good in vitro sealing capacity. ${ }^{23}$ However, Hammad et al..$^{24}$ analyzed fillings for the presence of gaps and voids in canals obturated with gutta-percha, Resilon, Endorez, or Guta-flow by lateral condensation. The gutta-percha presented the best results, while the canals obturated with Resilon presented greater indi-

\section{References}

1. Schilder H. Filling root canals in three dimensions. Dent Clin North Am. 1967 Nov:723-44.

2. Rubach WC, Mitchell DF. Periodontal disease, accessory canals and pulppathosis. J Periodontol. 1965 Jan-Feb;36:34-8.

3. De Deus QD. Frequency, location, and direction of the lateral, secondary, and accessory canals. J Endod. 1975 Nov;1(11):361-6.

4. Venturi M, Di Lenarda R, Prati C, Breschi L. An in vitro model to investigate filling of lateral canals. J Endod. 2005 Dec;31(12):877-81.

5. Weine FS. The enigma of the lateral canal. Dent Clin North Am. 1984 Oct;28(4):833-52.

6. Iqbal MK, Gartenberg J, Kratchman SI, Karabucak B, Bui B. The clinical significance and management of apical accessory canals in maxillary central incisors. J Am Dent Assoc. 2005 Mar;136(3):331-5; quiz 379-81.

7. Michelotto AL, Moura-Netto C, Araki AT, Akisue E, Moura AA, Sydney GB. In vitro analysis of thermocompaction time and gutta-percha type on quality of main canal and lateral canals filling. Braz Oral Res. 2010 Jul-Sep;24(3):290-5.

8. Tanomaru-Filho M, Sant'Anna Junior A, Berbert FL, Bosso R, Guerreiro-Tanomaru JM. Ability of gutta-percha and Resilon to fill simulated lateral canals by using the Obtura II system. J Endod. 2012 May;38(5):676-9.

9. Chandra SS, Shankar P, Indira R. Depth of penetration of four resin sealers into radicular dentinal tubules: a confocal microscopic study. J Endod. 2012 Oct;38(10):1412-6.

10. Shipper G, Ørstavik D, Teixeira FB, Trope M. An evaluation of microbial leakage in roots filled with a thermoplastic synthetic polymer-based root canal filling material (Resilon). J Endod. 2004 May;30(5):342-7.

11. Shipper G, Teixeira FB, Arnold RR, Trope M. Periapical inflammation after coronal microbial inoculation of dog roots filled with gutta-percha or resilon. J Endod. 2005 Feb;31(2):91-6.

12. Tay FR, Pashley DH, Williams MC, Raina R, Loushine RJ, Weller RN, et al. Susceptibility of a polycaprolactone-based root canal filling material to degradation. I. Alkaline hydrolysis. J Endod. 2005 Aug;31(8):593-8. ces of voids and gaps. Thus, the use of the Resilon Real Seal system for lateral root canals should be limited to thermoplastic obturation techniques.

\section{Conclusion}

The Resilon Real Seal system achieves deeper penetration into lateral root canals when associated with thermoplastic obturation techniques.

13. Miner MR, Berzins DW, Bahcall JK. A comparison of thermal properties between gutta-percha and a synthetic polymer based root canal filling material (Resilon). J Endod. 2006 Jul;32(7):683-6.

14. Karabucak B, Kim A, Chen V, Iqbal MK. The comparison of gutta-percha and Resilon penetration into lateral canals with different thermoplastic delivery systems. J Endod. 2008 Jul;34(7):847-9.

15. Reader CM, Himel VT, Germain LP, Hoen MM. Effect of three obturation techniques on the filling of lateral canals and the main canal. J Endod. 1993 Aug;19(8):404-8.

16. DuLac KA, Nielsen CJ, Tomazic TJ, Ferrillo PJ Jr, Hatton JF. Comparison of the obturation of lateral canals by six techniques. J Endod. 1999 May;25(5):376-80.

17. Tanomaru-Filho M, Silveira GF, Tanomaru JM, Bier CA. Evaluation of the thermoplasticity of different gutta-percha cones and Resilon. Aust Endod J. 2007 Apr;33(1):23-6.

18. Almeida JF, Gomes BP, Ferraz CC, Souza-Filho FJ, Zaia AA. Filling of artificial lateral canals and microleakage and flow of five endodontic sealers. Int Endod J. 2007 Sep;40(9):692-9.

19. Karr NA, Baumgartner JC, Marshall JG. A comparison of gutta-percha and Resilon in the obturation of lateral grooves and depressions. J Endod. 2007 Jun;33(6):749-52.

20. Tanomaru-Filho M, Sant'anna-Junior A, Bosso R, GuerreiroTanomaru JM. Effectiveness of gutta-percha and Resilon in filling lateral root canals using the Obtura II system. Braz Oral Res. 2011 May-Jun;25(3):205-9.

21. Epley SR, Fleischman J, Hartwell G, Cicalese C. Completeness of root canal obturations: Epiphany techniques versus gutta-percha techniques. J Endod. 2006 Jun;32(6):541-4.

22. Herbert J, Bruder M, Braunsteiner J, Altenburger MJ, Wrbas KT. Apical quality and adaptation of Resilon, EndoREZ, and Guttaflow root canal fillings in combination with a noncompaction technique. J Endod. 2009 Feb;35(2):261-4.

23. Oddoni PG, Mello I, Coil JM, Antoniazzi JH. Coronal and apical leakage analysis of two different root canal obturation systems. Braz Oral Res. 2008 Jul-Sep;22(3):211-5.

24. Hammad M, Qualtrough A, Silikas N. Evaluation of root canal obturation: a three-dimensional in vitro study. J Endod. 2009 Apr;35(4):541-4. 